\title{
Two-Photon Fabrication of Three-Dimensional Metallic Nanostructures for Plasmonic Metamaterials
}

\author{
Atsushi ISHIKAWA ${ }^{1}$ and Takuo TANAKA ${ }^{1,2}$ \\ 1- Metamaterials Laboratory, RIKEN, 2-1 Hirosawa, Wako, Saitama 351-0198, Japan \\ 2- Research Inst. for Electronic Science, Hokkaido University, Sapporo, Hokkaido 001-0020, Japan \\ E-mail: a-ishikawa@riken.jp and t-tanaka@riken.jp
}

\begin{abstract}
We propose a novel laser fabrication technique based on two-photon-induced reduction of metal ions for 3D metallic micro/nanostructures. Electrically conductive silver nanostructures with arbitrary 3D shape are demonstrated. By introducing dopant surfactant molecules, which prohibit unwanted metal growth, we demonstrate considerable improvement of the reduction properties to achieve a spatial resolution down to $120 \mathrm{~nm}$. Plasmonic metamaterials fabricated using this technique exhibit magnetic resonances with negative permeability at far-infrared frequencies.
\end{abstract}

DOI:10.2961/jlmn.2012.01.0002

Keywords: Laser fabrication, Two-photon absorption, Photoreduction of metal ions, 3D metallic nanostructures, Plasmonic metamaterials

\section{Introduction}

Plasmonic metamaterials has recently attracted much interest from researchers by introducing a new paradigm in electromagnetism. Metamaterial is a new class of artificial materials made of three-dimensional (3D) metallic nanostructures. Electromagnetic responses of the metamaterial are specified by its internal nanostructures, not by the composition. Therefore, by engineering the nanostructures, we are able to create unprecedented optical functionalities at will, even optical magnetism and negative index of refraction, which are never provided by natural materials [1-5].

There have recently been a number of reports on the realization of metamaterials by using lithography-based nanofabrication technologies, bringing various new optical phenomena into reality. However, such metamaterials demonstrated so far are mostly two-dimensional (2D) planar structures with strong anisotropy, thus their optical functionalities are extremely limited. This is due to lack of the ability to make arbitrary $3 \mathrm{D}$ metallic nanostructures in such conventional technologies, and the realization of 3D isotropic metamaterials remains a major challenge [6].

In this paper, we propose a novel laser fabrication technique based on two-photon-induced reduction of metal complex ions to realize 3D metallic micro/nanostructures. Electrically conductive silver nanostructures with arbitrary 3D shape are demonstrated. Using this technique, we fabricated plasmonic metamaterials that exhibit magnetic resonances with negative permeability at far-infrared frequencies.

\section{Two-photon fabrication of 3D metallic nanostruc- tures}

To realize the fabrication resolution in three dimensions, we employed the process of two-photon absorption (TPA), which has recently been investigated and widely used in various applications, such as fluorescence microscopy, optical data storage, and lithographic fabrication [7-10].
Since light absorption in the TPA process is highly localized at the focal point, it is possible to confine successive chemical or physical reactions in a small volume with high spatial resolution in three dimensions. By utilizing this unique property of the TPA process, we have recently proposed a novel fabrication technique based on two-photoninduced reduction of metal complex ions to realize 3D metallic micro/nanostructures [11-13].

For all experimental results reported here, a modelocked Ti:Sapphire laser system (Spectra-Physics, Tsunami) was used as a light source. The center wavelength, pulse width, and repetition rate are $800 \mathrm{~nm}, 80 \mathrm{fs}$, and 82 $\mathrm{MHz}$, respectively. The beam from the laser system was introduced into an inverted microscope (Olympus, IX71) and tightly focused into a host material including metal complex ions on a glass cover slip with an oil-immersion objective lens $(60 \times, \mathrm{NA}=1.42)$. In the fabrication process, a metal ion absorbs two photons simultaneously, being photo-reduced into metal nanoparticles. Since the TPA process occurs only at the focal point where the photon density is high enough, 3D fabrication below the diffraction limit can be achieved. The focused laser beam was then scanned two-dimensionally ( $x-y$ scanning) with twoaxis galvanometer mirrors. The focused laser beam was also scanned in the longitudinal direction (z scanning) by translating the objective lens with a computer-controlled motor stage installed in the microscope (Chuo Precision Industrial, MSS-FU). Since the laser beam scanning area was limited by the field view of the objective lens, which is up to about $300 \mu \mathrm{m}$ in diameter, the sample was also mounted on an $x-y$ translation stage in order to extend the fabrication area.

As a photoreactive medium, we initially use silver-ion solution, which was prepared by mixing $0.2 \mathrm{~mol} / 1$ silver nitrate $\left(\mathrm{AgNO}_{3}\right)$ aqueous solution with $0.01 \mathrm{wt} \%$ Coumarin 440 (Exciton Inc.) ethanol solution in a $1: 1$ volume ratio at room temperature. As shown in Figure 1, the solution had peak absorption and fluorescence wavelengths of 350 
$\mathrm{nm}$ and $430 \mathrm{~nm}$ due to the Coumarin 440. Since the $\mathrm{AgNO}_{3}$ aqueous solution without the dye is originally colorless, suggesting that its absorption cross-section is very small at visible wavelengths, incident light of $800 \mathrm{~nm}$ in wavelength is mainly absorbed by Coumarin 440 through the TPA process.

Figure 2 shows the basic scheme of the reduction of silver ions through the photoinduced electron transfer from the dye molecules to silver ions [14]. The electron transfer (ET) from the LUMO level of the excited dye to silver ions leads to the production of silver atoms. This photosensitized reduction process allowed a low laser power to trigger the reduction of silver ions, and sub-diffraction-limit fabrication was realized while suppressing local heating. Some of electrons, which once meet silver ions, will undergo charge recombination (CR) with the dye and sacrificial donor, and this $\mathrm{CR}$ sets the upper limitation of production rate of silver atoms. The ET from the sacrificial donor to the HOMO level regenerates the neutral state of the dye, allowing further absorption of photons and production of silver atoms. Inside the laser spot, silver poly-crystal grows continuously through these sequential reactions.

Figures 3(a) and 3(b) are 2D/3D silver microstructures fabricated in the silver-ion solution using this process.

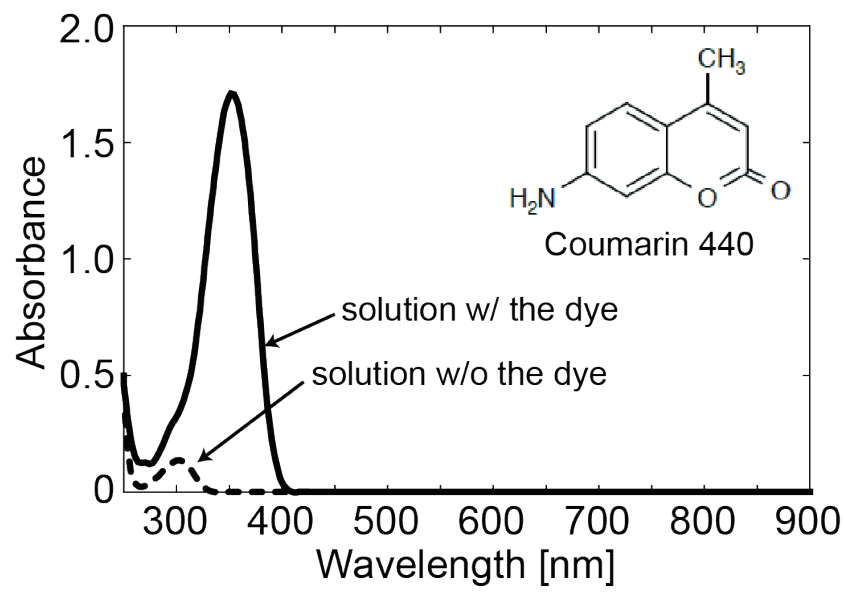

Fig. 1 Absorption spectra of $0.2 \mathrm{~mol} / 1 \mathrm{AgNO}_{3}$ aqueous solution mixed with $0.01 \mathrm{wt} \%$ Coumarin 440 ethanol solution and ethanol in a $1: 1$ volume ratio, measured with an absorption quartz cell with a thickness of $1 \mathrm{~mm}$. The inset is molecular structure of Coumarin 440.

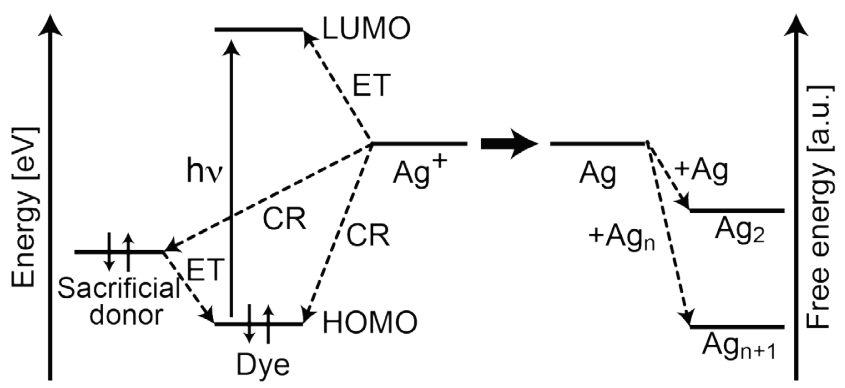

Fig. 2 Basic scheme of photoinduced electron transfer from the dye molecules to silver ions for the reduction of silver ions. Symbols denote: $\mathrm{Ag}^{+}$, silver ions; $\mathrm{Ag}$, silver atom; ET, the electron transfer; $\mathrm{CR}$, charge recombination; HOMO, the highest occupied molecular orbital; LUMO, the lowest unoccupied molecular orbital.
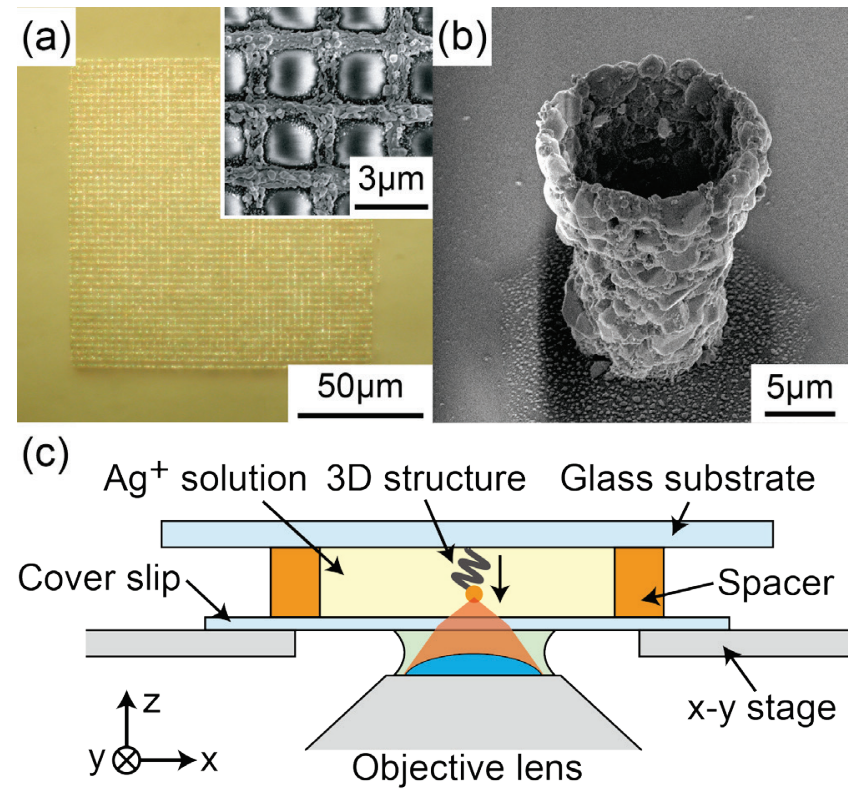

Fig. 3 (a) Optical microscope image of $2 \mathrm{D}$ silver mesh with a total size of $120 \times 120 \mu \mathrm{m}^{2}$. The minimum width of the wire was $400 \mathrm{~nm}$ [the inset SEM image in (a)]. (b) SEM image of a freestanding 3D silver cup on a substrate. The height and diameter of the cup were $20 \mu \mathrm{m}$ and $15 \mu \mathrm{m}$, respectively. (c) Experimental setup for the upside-down fabrication of 3D structures.

During the fabrication, quenching of the fluorescence of the dye was clearly observed, indicating the photosensitized reduction occurred. Fig. 3(a) is an optical microscope image of 2D silver mesh with a total size of $120 \times 120 \mu \mathrm{m}^{2}$ The total exposure time, laser power, and scan speed were $12.15 \mathrm{~s}, 13.66 \mathrm{~mW}$, and $50 \mu \mathrm{m} / \mathrm{s}$, respectively. As seen in the inset SEM image of Fig. 3(a), the minimum wire width of $400 \mathrm{~nm}$ was achieved under this condition. In addition to the improved reduction properties and spatial resolution based on the photosensitized reduction process, the electrical properties of the wires were at least as good as those fabricated without using a dye [11]. The averaged resistivity of $5.30 \times 10^{-8} \Omega \mathrm{m}$ was obtained in these wires, and this value is only 3.3 times larger than that of bulk silver (1.62 $\left.\mathrm{x} 10^{-8} \Omega \mathrm{m}\right)$. The discrepancy is due to the roughness and oxidization or sulfurization of the silver wire surface. Since the solution with the dye was highly reactive, it should be possible to fabricate even larger structures in less time by combining our method with a laser beam interference or micro-lens array technique $[15,16]$.

We also demonstrated a free-standing 3D silver cup on a substrate as shown in Fig. 3(b). For the fabrication of 3D structures, we introduced upside-down experimental setup [Fig. 3(c)], since the deposited silver is not transparent at $800 \mathrm{~nm}$ in wavelength. The formation mechanism of 3D structures is based on crystal growth rather than aggregation of the particles; otherwise the produced silver particles should fall down without being deposited in this configuration. This is also supported by the observation of the surface roughness in Fig. 3(b), which consists of silver polycrystal structures. Since the photoreductive reaction far from the substrate is susceptible to the conditions in the solution (convection, turbulence, and so on), its efficiency is somewhat low and variable as compared to that at the 
interface between the substrate and the solution. However, the high reactivity of the solution enabled us to build up solid silver structures efficiently and repeatedly. The strength of the structures produced was sufficient to fabricate a silver cup that could independently stand on the substrate, as seen in Fig. 3(b).

\section{Improvement of the reduction properties using do- pant surfactant molecules}

Since the feature size of plasmonic metamaterials operating at optical frequencies is typically on the order of 100 $\mathrm{nm}$ or even smaller, further improvement of the reduction properties, especially in the resolution, is highly anticipated. In addition to the local heating, the major problem that inhibited the nanoscale resolution is the unwanted crystal growth during laser irradiation. Therefore, the main issue to gain the nanometer scale depends on a way to avoid this unwanted crystal growth and produce smaller nanoparticles to serve as building blocks.

To this end, we introduced a dopant surfactant molecule, a nitrogen-atomcontaining alkyl carboxylate (ndecanoylsarcosine sodium, NDSS) as a crystal growth inhibitor into silver-ion solution [17,18]. Figure 4 shows the absorption spectra of $0.05 \mathrm{~mol} / \mathrm{l}$ diammine silver ions (DSI) aqueous solution mixed with $0.099 \mathrm{~mol} / 1 \mathrm{NDSS}$ aqueous solution. For the reference in Fig. 4, we also show the absorption spectra of pure DSI and pure NDSS aqueous solutions. An absorption band with the peak at $302 \mathrm{~nm}$ is clearly visible in the spectra for both the pure DSI and the mixture of DSI and NDSS solutions, but not in that for pure NDSS. This absorption band originates from the DSI itself. In all spectra, the remarkable other absorption band is not observed at the laser wavelength of $800 \mathrm{~nm}$. This implies that the photo-induced reduction of complex silver ions was associated with exciting the chemicals by the TPA process.

Figure 5 shows 2D/3D silver nanostructures fabricated in the silver-ion solution with NDSS. Fig. 5(a) is a silver wire fabricated with a laser power of $0.87 \mathrm{~mW}$ and a scan speed of $6 \mu \mathrm{m} / \mathrm{s}$. The formation mechanism of silver structures is completely different from the previous case without

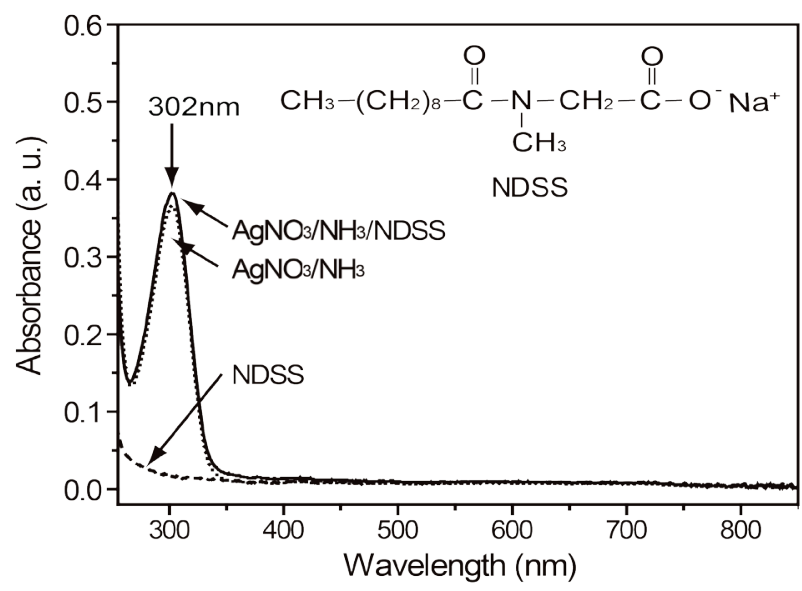

Fig. 4 Absorption spectra of $0.05 \mathrm{~mol} / 1$ DSI aqueous solution mixed with $0.099 \mathrm{~mol} / 1 \mathrm{NDSS}$ aqueous solution (solid line), pure $0.05 \mathrm{~mol} / 1 \mathrm{DSI}$ aqueous solution (dotted line), and pure $0.4 \mathrm{~mol} / 1$ NDSS aqueous solution (dashed line). The inset is molecular structure of NDSS.

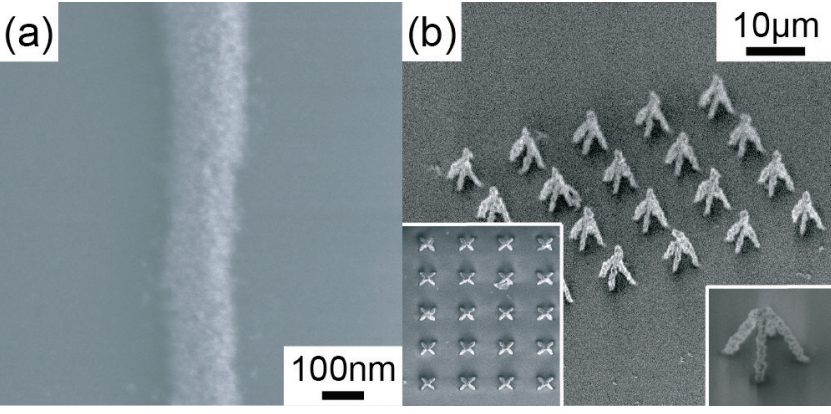

Fig. 5 SEM images of (a) a $2 \mathrm{D}$ silver wire with the minimum width of $120 \mathrm{~nm}$ and (b) free-standing 3D silver pyramids on a substrate. The height and the angle for each edge relative to the substrate were $5 \mu \mathrm{m}$ and $60^{\circ}$. The insets are the top view of the pyramids (left) and its magnified image (right).

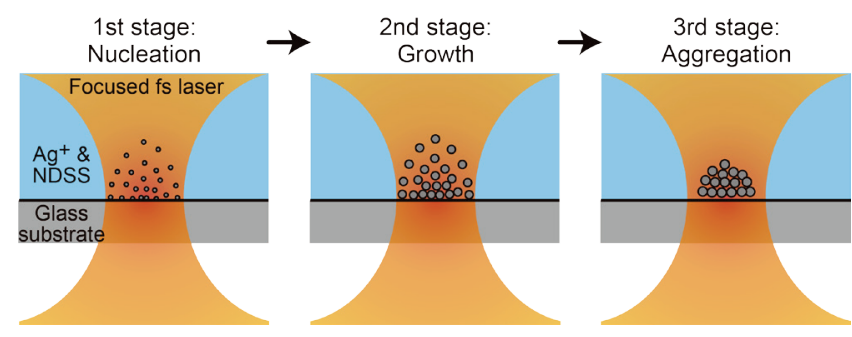

Fig. 6 Basic scheme of the formation of silver structures from the silver-ion solution with NDSS. The process starts from the first stage where silver ions are photo-reduced, creating silver seeds at the focal point. At the second stage, the silver seeds grow up to particles with uniform size and shape. At the third stage, aggregation of the particles leads to the formation of silver structures.

NDSS. Figure 6 shows the basic scheme of the formation mechanism of the structures in the surfactant-assisted photoreduction process. At the first stage just after the laser irradiation, NDSS molecules immediately cover the surface of the silver particles after the nucleation process and then eliminate further crystal growth and decrease the particle size down to around $20 \mathrm{~nm}$. These growth-suppressed particles densely aggregate to compose silver patterns. The concentration of particles is higher at the center of the focused laser spot, since there is a higher nucleation probability associated with the higher laser intensity. At the same time, this higher laser intensity also helps to break the surfactant layer surrounding the particles, which in turn enhances the adhesion between the particles. These two conditions lead to the aggregation of silver particles directed to the center of the laser beam. As a result, we have successfully improved the spatial resolution down to $120 \mathrm{~nm}$, as shown in Fig. 5(a).

Fig. 5(b) demonstrates free-standing 3D silver pyramids fabricated with a laser power of $1.3 \mathrm{~mW}$ and a scanning speed of $2.5 \mu \mathrm{m} / \mathrm{s}$. These silver pyramids structures were strong enough to resist the surface tension in the washing process, which demonstrates that the silver particles were closely combined. The right inset in Fig. 5(b) reveals that the height was $5 \mu \mathrm{m}$ and the angle for each leg relative to the substrate was $60^{\circ}$. Consequently, the direct photoreduction of complex metal ions with the help of surfactant molecules could lead to 3D metal nanostructures with the resolution exceeding the diffraction limit of light. 
4. Magnetic metamaterials at far-infrared frequencies

Using the fabrication technique presented here, we have fabricated plasmonic metamaterials operating at farinfrared frequencies [19]. Figures 7(a) and 7(b) show a photograph and its SEM image of a 2D metamaterial consists of silver rod pair array. The unit-cell dimensions, $a_{x}$ and $a_{y}$, were $15 \mu \mathrm{m}$, the rod length, $l$, was $10 \mu \mathrm{m}$, the rod width, $w$, was $1.5 \mu \mathrm{m}$, and the distance between two rods, $g$, was $4 \mu \mathrm{m}$; the total size of the sample was $3 \times 3 \mathrm{~mm}^{2}$. All silver structures were fabricated on a z-cut quartz substrate with a thickness of $150 \mu \mathrm{m}$. As shown in Fig. 7(c), the rod pair structure has the geometrical inductance (L) and capacitance $(\mathrm{C})$, which respectively comes from the rectangular area sandwiched between two rods and the gap between two rods; it acts as an LC-resonant circuit coupled with the magnetic field. When the incident $s$ (TE)-polarized light passes through the sample at the incident angle of $\theta$, as shown in Fig. 7(d), the magnetic field perpendicular to the plane including two rods is $H \sin \theta$, where $H$ is the magnetic field of the incident light. Therefore, by increasing the incident angle at which the larger magnetic field is introduced, strong magnetic excitation of the magnetic resonances can be obtained. In addition to the rod pair array, we also fabricated a single (unpaired) rod array [the inset in Fig. 8(a)], which doesn't form an LC-resonant circuit, to confirm that the magnetic responses were observed only in the rod pair array. The single rod array had the same rod-

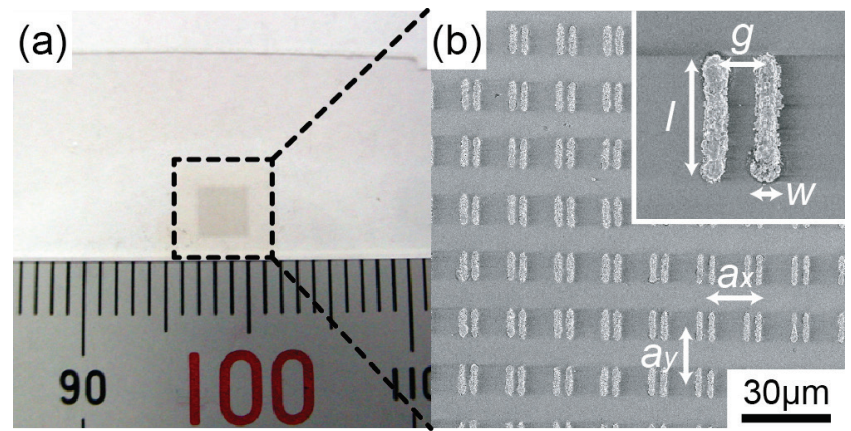

(c)

(d) Al aperture

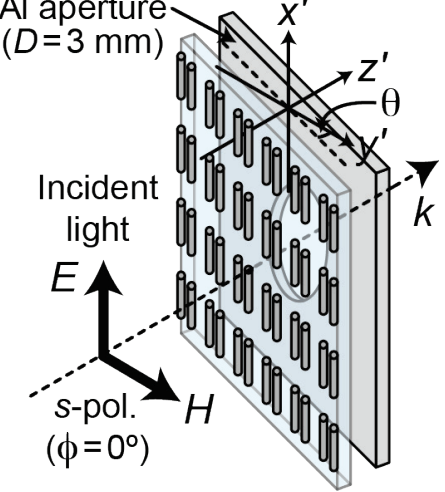

Fig. 7 (a) $3 \times 3 \mathrm{~mm}^{2}$ silver rod array fabricated on a quartz substrate and (b) its SEM image. The unit-cell dimensions, $a_{x}$ and $a_{y}$ were $15 \mu \mathrm{m}$, the rod length, $l$, was $10 \mu \mathrm{m}$, the rod width, $w$, was $1.5 \mu \mathrm{m}$, and the distance between two rods, $g$, was $4 \mu \mathrm{m}$. (c) The rod pair structure acts as an LC-resonant circuit, and the induced current $(\mathrm{j})$ is generated by the magnetic field perpendicular to the plane including two rods $(\mathrm{H} \sin \theta)$. (d) Optical setup for measuring the magnetically excited magnetic responses of the rod pair array by changing the incident angle $(\theta)$ of $s$-polarized light.
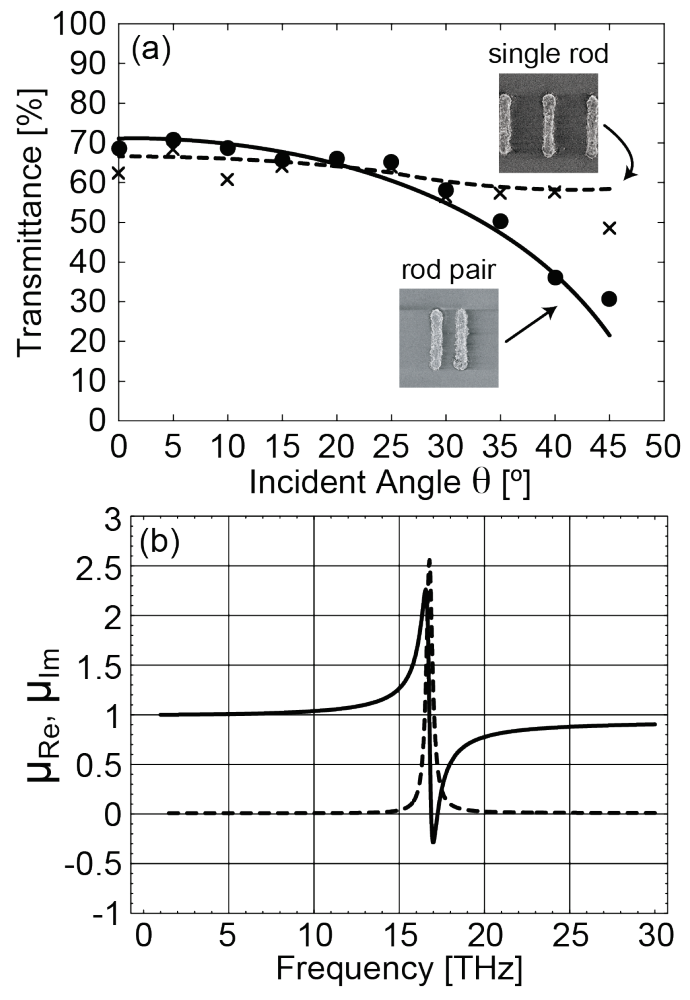

Fig. 8 (a) Incident angle dependencies of the transmittances of the rod pair array and the single rod array measured at $18 \mathrm{THz}$. The insets are SEM images of the corresponding unit-cell structures. (b) The numerically simulated real and imaginary parts of the effective permeability ( $\mu_{\mathrm{Re}}$ : solid line and $\mu_{\mathrm{Im}}$ : dotted line) of the silver rod pair array. The magnetic resonance response in $\mu$ is clearly observed around the resonant frequency of $16.77 \mathrm{THz}$, which was in good agreement with the experimental results.

filling factor as that of the rod pair array; therefore, we could compensate for the influence of the electric interactions between the structures and light by comparing the transmission spectra of the two structures.

Figure 8(a) shows the incident angle dependencies of the transmittances of the rod pair array and the single rod array measured at $18 \mathrm{THz}$. All transmission spectra were measured by using a Fourier-transform infrared spectrometer (FT-IR; JASCO, FT/IR-6300FV). The measurement spectral range was from 0.9 to $20.4 \mathrm{THz}$, with a resolution of $0.1 \mathrm{THz}$. An aluminum aperture with a diameter of 3 $\mathrm{mm}$ was installed between the sample and the photodetector to improve the signal-to-noise ratio of the detected signal. When the incident angle increased, the transmittance of the single rod array did not change and remained almost constant at $65 \%$, indicating that the single rod array did not interact with the magnetic field. On the other hand, the transmittance of the rod pair array uniformly decreased from $70 \%$ to $20 \%$ as the incident angle increased. Since the increase of the incident angle leads to an increase of the magnetic field that interacts with the rod pair structure, this result directly proves that magnetically excited magnetic resonances occurred in the rod pair array.

To investigate this experimental result further, we also performed a corresponding numerical simulation of the magnetic responses of the rod pair array [1-3]. Fig. 8(b) shows the numerically simulated real and imaginary parts of the effective permeability $\left(\mu_{\mathrm{Re}}\right.$ and $\left.\mu_{\mathrm{Im}}\right)$ of the silver rod 
pair array. In the calculation, we used the damping constant $\gamma=1.06 \times 10^{14} \mathrm{~s}^{-1}$, which is 3.3 times larger than that of bulk silver $\left(\gamma=3.23 \times 10^{13} \mathrm{~s}^{-1}\right)$ to consider the optical quality of the fabricated silver structure by using our technique [20]. In Fig. 8(b), the magnetic resonance response in $\mu$ is clearly observed around the resonant frequency of 16.77 THz, and the value of $\mu_{\mathrm{Re}}$ changes from -0.29 to 2.26 . Since $\mu_{\mathrm{Im}}$ has a finite value around the resonant frequency, the absorption of the incident light should be observed; this is in good agreement with the experimental results and the above discussion.

The proposed laser fabrication technique, in principle, can be applied to 3D plasmonic metamaterials by replacing aqueous solution with an appropriate host material to support and stack metallic nanostructures three-dimensionally. To demonstrate this idea, we used a trivalent gold ion $\left(\mathrm{Au}^{3+}\right)$ doped Poly(methyl methacrylate) (PMMA) film as a photoreactive medium and fabricated $2 \mathrm{D}$ split-ring resonator array inside the film. Figure 9 is an optical microscope image of the exposed PMMA film. Although gold microstructures are supported inside the PMMA, they consist of closely-aggregated gold particles, which may not be electrically connected each other. This is due to the limited gold-ion mobility inside the PMMA, which is not as high as that in aqueous solution; therefore, during the fabrication, the concentration of gold ions within the laser spot cannot be high enough to form solid gold patterns. For this fabrication technique, a host medium has to be transparent at $800 \mathrm{~nm}$ in wavelength, but also at the metamaterial's operating wavelength for the subsequent optical measurements. Therefore, a comprehensive survey of suitable host materials that have high ion mobility and transparent window at specific wavelengths is highly anticipated for the realization of 3D metamaterials. Mass production would be another issue for such 3D metamaterials, but may be solved by combining other promising techniques, such as a laser beam interference or micro-lens array $[15,16]$.

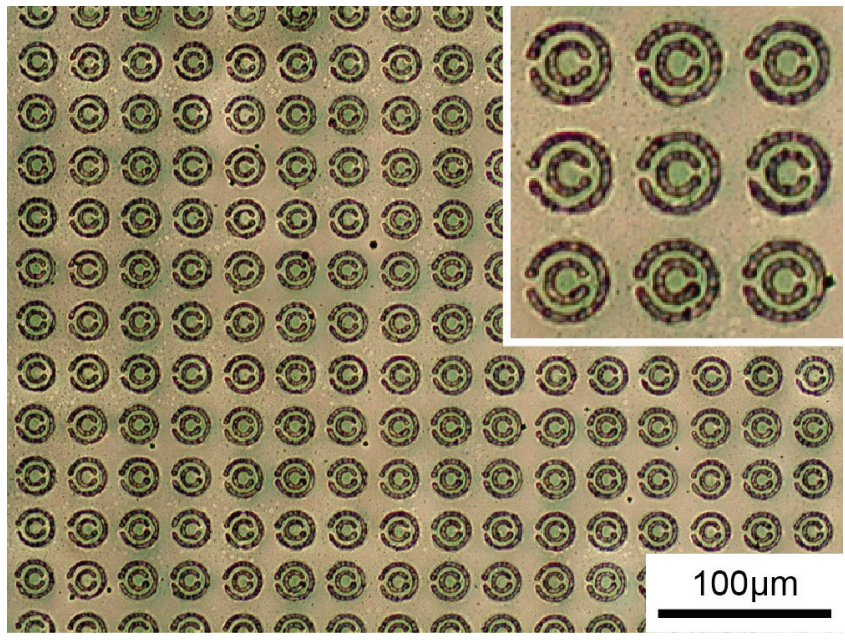

Fig. 9 Optical microscope image of gold split-ring resonator array fabricated in $\mathrm{Au}^{3+}$ doped PMMA film.

\section{Conclusions}

We have proposed a novel laser fabrication technique based on two-photon-induced reduction of metal ions to realize 3D metallic micro/nanostructures. Electrically conductive silver nanostructures with arbitrary 3D shape are demonstrated. By introducing dopant surfactant molecules, which prohibit unwanted metal growth, we demonstrate considerable improvement of the reduction properties to achieve a spatial resolution down to $120 \mathrm{~nm}$. Using the proposed technique, we have fabricated plasmonic metamaterials that exhibit magnetic resonances with negative permeability at far-infrared frequencies.

\section{Acknowledgments}

A. Ishikawa acknowledges support from the RIKEN Special Postdoctoral Researchers Program. The results of calculations were performed by using the RIKEN Super Combined Cluste (RSCC). This work was partly supported by JST PRESTO and by a Grant-in-Aid for Scientific Research on Innovative Areas (No. 22109006) from the Ministry of Education, Culture, Sports, Science and Technology (MEXT), Japan.

\section{References}

[1] A. Ishikawa, T. Tanaka, and S. Kawata, Phys. Rev. Lett. 95, 237401 (2005).

[2] A. Ishikawa and T. Tanaka, Opt. Commun. 258, 300 (2006).

[3] A. Ishikawa, T. Tanaka, and S. Kawata, J. Opt. Soc. Am. B 24, 510 (2007).

[4] T. Tanaka, A. Ishikawa, and S. Kawata, Phys. Rev. B 73, 125423 (2006).

[5] A. Ishikawa, S. Zhang, D. A. Genov, G. Bartal, and X. Zhang, Phys. Rev. Lett. 102, 043904 (2009).

[6] C. M. Soukoulis and M. Wegener, Nat. Photonics 5, 523 (2011).

[7] W. Denk, J. H. Strickler, and W. W. Webb, Science 248, 73 (1990).

[8] D. A. Parthenopoulos and P. M. Rentzepis, Science 245, 843 (1989).

[9] P.-W. Wu et al., Adv. Mater. 12, 1438 (2000).

[10] W. Zhou et al., Science 296, 1106 (2002).

[11] T. Tanaka, A. Ishikawa, and S. Kawata, Appl. Phys. Lett. 88, 81107 (2006).

[12] A. Ishikawa, T. Tanaka, and S. Kawata, Appl. Phys. Lett. 89, 113102 (2006).

[13] T. Tanaka, J. Laser Micro/Nanoeng. 3, 152 (2008).

[14] J. F. Hamilton, Adv. Phys. 37, 359 (1988).

[15]F. Formanek, N. Takeyasu, T. Tanaka, K. Chiyoda, A. Ishikawa, and S. Kawata, Opt. Express 14, 800 (2006).

[16]F. Formanek, N. Takeyasu, K. Chiyoda, T. Tanaka, A. Ishikawa, and S. Kawata, Appl. Phys. Lett. 88, 83110 (2006).

[17] Y.-Y. Cao et al., Small 5, 1144 (2009).

[18] T. Kempa et al., Plasmonics 1, 45 (2006).

[19] A. Ishikawa, T. Tanaka, and S. Kawata, Appl. Phys. Lett. 91, 113118 (2007).

[20] P. B. Johnson and R.W. Christy, Phys. Rev. B 6, 4370 (1972).

(Received: June 7, 2011, Accepted: December 9, 2011) 